\title{
LPA $_{3}$-mediated lysophosphatidic acid signalling in implantation
}

\section{and embryo spacing}

\author{
Xiaoqin Ye $\mathrm{e}^{1,6}$, Kotaro Hama $^{2,6}$, James J.A. Contos ${ }^{3}$, Brigitte Anliker ${ }^{1}$, Aska Inoue ${ }^{2}$, Michael \\ K. Skinner ${ }^{4}$, Hiroshi Suzuki ${ }^{5}$, Tomokazu Amano ${ }^{5}$, Grace Kennedy ${ }^{1}$, Hiroyuki Arai ${ }^{2}$, Junken \\ Aoki $^{2}$, and Jerold Chun ${ }^{1}$ \\ 1 Department of Molecular Biology, Helen L. Dorris Child and Adolescent Neuro-psychiatric Disorder \\ Institute, The Scripps Research Institute, 10550 North Torrey Pines Road, La Jolla, CA 92037, USA. \\ 2 Graduate School of Pharmaceutical Sciences, University of Tokyo, 7-3-1, Hongo, Bunkyo-ku, Tokyo, \\ 113-0033, Japan. \\ 3 Fred Hutchinson Cancer Research Center, 1100 Fairview Avenue North, Seattle, WA 98109-1024. \\ 4 Center for Reproductive Biology, School of Molecular Bioscience, Washington State University, Pullman, \\ WA 99164-4231, USA. \\ 5 Developmental and Medical Technology (Sankyo), Department of Medicine, University of Tokyo, 7-3-1, \\ Hongo, Bunkyo-ku, Tokyo, 113-0033, Japan
}

\section{Abstract}

Every successful pregnancy requires proper embryo implantation. Low implantation rate is a major problem during infertility treatments using assisted reproductive technologies (ART) ${ }^{1}$. Here we report a new molecular influence on implantation through the lysophosphatidic acid (LPA) receptor $\mathrm{LPA}_{3}{ }^{2-4}$. Targeted deletion of $\mathrm{LPA}_{3}$ in mice resulted in significantly reduced litter size, which could be attributed to delayed implantation and altered embryo spacing. These two events led to delayed embryonic development, hypertrophic placentas shared by multiple embryos, and embryonic death. An enzyme demonstrated to influence implantation, cyclooxygenase-2 (COX-2) 5 , was downregulated in $\mathrm{LPA}_{3}$-deficient uteri during preimplantation. Down regulation of COX-2 led to reduced levels of prostaglandins that are critical for implantation ${ }^{1}$. Exogenous administration of the prostaglandins $\mathrm{PGE}_{2}$ and cPGI into $\mathrm{LPA}_{3}$-deficient females rescued delayed implantation but did not rescue defects in embryo spacing. These data identify $\mathrm{LPA}_{3}$ receptor-mediated signalling as a new influence on implantation and further indicate linkage between LPA signalling and prostaglandin biosynthesis.

\begin{abstract}
Multiple factors can adversely affect successful pregnancy. Two of these factors are failed synchronization between embryonic and endometrial development during implantation and occurrence of multiple gestations (especially monochorionic gestation), which can result in fetal demise ${ }^{1,6-9}$. These factors are particularly important for the clinical success and efficacy of ART. One molecular factor that has been previously implicated in female reproduction is the small, bioactive phospholipid LPA ${ }^{10}$. LPA has a range of influences that are mediated by at least four $\mathrm{G}$ protein-coupled receptors, $\mathrm{LPA}_{1-4}{ }^{2}$. Deletion of $\mathrm{LPA}_{1}$ and $\mathrm{LPA}_{2}$ in mice revealed roles for these receptors in neural development, craniofacial formation, neuropathic pain, and altered cellular signalling, but without obvious effects on female reproduction ${ }^{11-}$
\end{abstract}

\footnotetext{
Correspondence and requests for materials should be addressed to J. Chun (e-mail:jchun@ @cripps.edu)..

These authors contributed equally to the work.

Supplementary Information accompanies the paper on http://www.nature.com/nature.

Competing Interests statement The authors declare that they have no competing financial interests.
} 
14. These results suggested that LPA signalling in female reproduction might be mediated by other LPA receptors including $\mathrm{LPA}_{3}$ (formerly known as Edg7) ${ }^{3,4}, \mathrm{LPA}_{4}{ }^{15}$, unidentified LPA receptor(s), or possibly non-receptor pathways. Towards identifying LPA-dependent mechanisms affecting reproduction, we targeted $\mathrm{LPA}_{3}$ for deletion, a receptor with distinct signalling properties and a preference for unsaturated LPA species ${ }^{2-4}$.

Functional deletion of $\mathrm{LPA}_{3}$ was achieved by replacing a fragment covering the untranslated region and the start codon in exon 2 with a neomycin-resistance gene in reverse orientation in R1 embryonic stem cells (supplementary Fig. S1). The LPA ${ }_{3}$-deficient mice were born with normal Mendelian frequency without sexual bias (supplementary Table S1), and appeared grossly normal (data not shown). However, $\mathrm{LPA}_{3}$-deficient females produced litter sizes of less than 50\% compared to that from wild-type (WT) and $\mathrm{LPA}_{3}$ heterozygote (Het) controls (supplementary Table S2), and showed a statistically significant prolongation of pregnancy (20.9 \pm 0.5 days, vs. $19.4 \pm 0.7$ days in $\mathrm{WT} / \mathrm{Het}$ controls, $\mathrm{P}<0.05$ ). These phenotypes were independent of stud genotype, indicating defects in female reproduction.

Towards determining whether $\mathrm{LPA}_{3}$ deletion might directly affect the female reproductive system, expression patterns of $\mathrm{LPA}_{3}$ mRNA were assessed using RT-PCR and in situ hybridization. By RT-PCR, $\mathrm{LPA}_{3}$ mRNA was detected in oviduct, placenta and uterus but not in ovary and eggs (unfertilized eggs and fertilized eggs from one cell to preimplantation blastocyst, data not shown). Within the uterus, $\mathrm{LPA}_{3}$ mRNA expression was up-regulated during postnatal development and varied during the estrous cycle (supplementary Fig. S3a, S3b). Strikingly, $\mathrm{LPA}_{3} \mathrm{mRNA}$ levels increased during early pregnancy, peaking around embryonic day 3.5 (E3.5) then returning to basal levels from E4.5 through the end of pregnancy (Fig. 1a). RT-PCR of microdissected E3.5 uterine tissue and in situ hybridization indicated that $\mathrm{LPA}_{3}$ mRNA expression was confined to the luminal endometrial epithelium at E3.5 (Fig. 1b, 1c, 1d, supplementary Fig. S3c). These data suggested that $\mathrm{LPA}_{3}$ loss-of-function resulting in reduced litter sizes could involve direct effects on the female reproductive system.

To explore this possibility, we examined major events in female reproduction, from ovulation through decidualization. No significant differences were observed in superovulation, fertilization, or decidualization between WT or Het controls and $\mathrm{LPA}_{3}$-deficient female littermates. No significant differences in blastocyst number or developmental stage, isolated from E3.5 uteri, were observed between controls and $\mathrm{LPA}_{3}$-deficient females. These data indicated no obvious defects in ovulation, ovum transportation, and blastocyst development in $\mathrm{LPA}_{3}$-deficient females (Fig. 1e, supplementary Fig. S4).

In notable contrast, embryo implantation studies identified clear phenotypic changes in LPA $_{3}$-deficient dames: delayed implantation and altered positioning/crowding of embryos. By E4.5, implantation sites identifiable by Evans blue labelling in control animals were absent in uteri of $\mathrm{LPA}_{3}$-deficient dames. Implantation sites became detectable at E5.5 in $\mathrm{LPA}_{3}$-deficient uteri (Fig. 1f, 1g) ${ }^{16}$. The number of pre-implantation blastocysts recovered from E4.5 LPA $3^{-}$ deficient uteri was comparable to that from E3.5 control and LPA 3 -deficient uteri (Fig. 1e, supplementary Fig. S4c), indicating that delayed implantation in $\mathrm{LPA}_{3}$-deficient uteri was ascribable to extra-embryonic influences of LPA signalling. In addition to delayed implantation, $\mathrm{LPA}_{3}$-deficient uteri had a reduced number of implantation sites compared to that in the control uteri in spite of the fact that comparable numbers of blastocysts were available for implantation. The implantation sites in the $\mathrm{LPA}_{3}$-deficient uteri were crowded/clustered in the uterine segments proximal to the cervix (Fig. 1g). This aberrant crowding of embryos in the $\mathrm{LPA}_{3}$-deficient uteri was further demonstrated by findings at later gestational stages. At E10.5, $44 \%$ of implantation sites in $\mathrm{LPA}_{3}$-deficient uteri contained 2-4 embryos (averaging 1.65 live embryos per implantation site) (Fig. 2a, 2b). At E18.5, 28\% of placentas were shared 
by $2-3$ embryos (Fig. 2c) and associated with placental hypertrophy (Fig. 2d, supplementary Fig. S5). These phenomena were never observed in controls.

In addition, embryos isolated from $\mathrm{LPA}_{3}$-deficient uteri (at E10.5 and E18.5) were always smaller than those from WT/Het controls at comparable ages (Fig. 3a, 3b, 3c), although newborns from $\mathrm{LPA}_{3}$-deficient females were, on average, heavier (Fig. 3c), possibly resulting from prolonged pregnancy and/or smaller litter size. The average number of live embryos per animal that could be isolated from $\mathrm{LPA}_{3}$-deficient females decreased following initial implantation (Fig. 3d). Delayed implantation and aberrant embryo spacing were thus associated with both delayed embryonic development and embryonic death, which could account for the reduced litter sizes produced by $\mathrm{LPA}_{3}$ deficiency.

To determine whether $\mathrm{LPA}_{3}$ loss in the embryo itself might contribute to the phenotypes, embryo transfer experiments were pursued. WT blastocysts were transferred to WT or $\mathrm{LPA}_{3^{-}}$

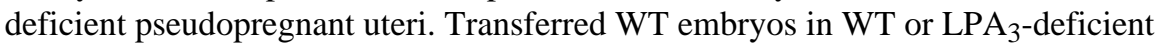
pseudopregnant uteri were phenotypically indistinguishable for implantation and development compared to embryos produced by natural matings in WT or LPA -deficient animals.

Similarly, when $\mathrm{LPA}_{3}$-deficient blastocysts were transferred to WT pseudopregnant uteri, no implantation or spacing abnormalities were observed (supplementary Fig. S6 and data not shown). WT and $\mathrm{LPA}_{3}$-deficient blastocysts had comparable implantation rates in WT pseudopregnant uteri. Considering the fact that no $\mathrm{LPA}_{3}$ mRNA was detected in WT preimplantation blastocysts, these results eliminate significant contributions of $\mathrm{LPA}_{3}$ via pre-/ post-implantation embryos, and indicate that maternal $\mathrm{LPA}_{3}$ signalling is responsible for the observed phenotypes.

The observed implantation phenotypes of $\mathrm{LPA}_{3}$-deficient mice were strikingly similar to that reported in rats and mice treated with indomethacin 17,18 , and mice deficient for cytosolic phospholipase $\mathrm{A}_{2 \alpha}\left(\mathrm{cPLA}_{2 \alpha}\right){ }^{19}$. Indomethacin is an inhibitor of cyclooxygenases ${ }^{20}$, which convert arachidonic acid (AA) to prostaglandin $\mathrm{H}_{2}\left(\mathrm{PGH}_{2}\right)$ in the biosynthesis of prostaglandins (PGs), while cPLA ${ }_{2 \alpha}$ is an important enzyme producing AA. Interestingly, COX-2-deficiency but not COX-1-deficiency in mice results in multiple female reproductive failures, including implantation defects 5,21 , although the precise phenotypes can be influenced by genetic background 22,23 . Moreover, $\mathrm{PGE}_{2}$ and cPGI (a stable analogue of $\mathrm{PGI}_{2}$ ) can partially correct implantation defects in both $\mathrm{cPLA}_{2 \alpha}$-deficient mice and COX-2deficient mice 19,24 . These data indicate that the $\mathrm{CPLA}_{2 \alpha}-\mathrm{AA}-\mathrm{COX}-\mathrm{PG}$ pathway is crucial for implantation ${ }^{1}$. In view of the phenotypic similarities between $\mathrm{LPA}_{3}$ deficiency and $\mathrm{cPLA}_{2 \alpha} / \mathrm{PG}$ deficiency, we hypothesized that $\mathrm{LPA}_{3}$ might converge on this signalling pathway.

Components of prostaglandin signalling were therefore examined in $\mathrm{LPA}_{3}$-deficient uteri. They include $\mathrm{cPLA}_{2 \alpha}, \mathrm{COX}-1$ and $\mathrm{COX}-2$, and prostaglandin $\mathrm{G}$ protein-coupled receptors $\mathrm{EP}_{1-4}$ and IP 25 , along with leukaemia inhibitory factor (LIF) and Hoxa-10, two key regulators in implantation ${ }^{1}$. Only COX-2 mRNA levels were significantly reduced in $\mathrm{LPA}_{3}$-deficient uteri (Fig. 4a, supplementary Fig. S7, and data not shown). Since COX-2 is a rate-limiting enzyme for PG biosynthesis, the suppression of COX-2 expression in E3.5 $\mathrm{LPA}_{3}$-deficient uteri resulted in reduced production of $\mathrm{PGE}_{2}$ and $\mathrm{PGI}_{2}$ (Fig. $4 \mathrm{~b}$ ), which may be inadequate for implantation that normally occurs around E4.0 18,26. To rescue this reduction in prostaglandins, we delivered exogenous $\mathrm{PGE}_{2}$ and cPGI to E3.5 $\mathrm{LPA}_{3}$-deficient females, a general approach previously reported ${ }^{19}$. Following PG exposure, significantly more $\mathrm{LPA}_{3}$ deficient females with on-time implantation were detected compared to vehicle-controls (Fig. $4 c, P=0.003)$. Notably, this rescue did not affect the uneven embryo spacing nor completely restore the reduction of implantation sites compared to WT controls (Fig. 4d). 
These findings identify LPA signalling as a new influence on embryo implantation, and are the first to link a lysophospholipid $\mathrm{G}$ protein-coupled receptor to prostaglandin biosynthesis, thereby influencing female fertility. As a class, lysophospholipid receptors represent a "drugable" target, as demonstrated by the compound FTY720 that is currently in phase III clinical trials for prevention of transplantation rejection 27 . This raises the possibility of creating medicines that influence implantation timing, a critical factor for in vitro fertilization 1,9 and reducing the increased incidence of multi-embryo gestations, especially monochorionic gestations that can result in fetal demise 6 . The reduced litter sizes observed in receptor-null mutants for another lysophosopholipid, sphingosine 1-phosphate, suggest that other lysophospholipid receptors may also influence mammalian reproduction through pharmacologically tractable mechanisms 28 .

\section{Materials and Methods}

\section{Quantitative RT-PCR}

Primers used were as described $11,12,29$. For amplification of COX-2, the following primers were used: forward, aagcgaggacctgggttca; reverse, aaggcgcagtttatgttgtctgt. Quantitative RTPCR was performed as described 29 . The transcript number of target genes was quantified and normalized against GAPDH or $\beta$-actin transcript number.

\section{In Situ Hybridization and Histology}

The animals were anesthetized with Halothane inhalation followed by cervical dislocation. In situ hybridization and histology were performed as described ${ }^{30}$. Sense and antisense DIGlabelled cRNA probes were generated using appropriate polymerases from a full-length murine $\mathrm{LPA}_{3} \mathrm{cDNA}$.

\section{Mating Study, Embryo Collection, and Localization of Implantation Sites}

All the mice used in this study were mixed background (129/SvJ and C57BL/6). Since no difference was observed in all the parameters examined between WT and Het females (Table S2, supplementary Fig. S8a, S8b, and data not shown), females with either one or both WT and Het genotypes were used as controls. Females were naturally mated with WT stud males. The day a plug found was designated as E0.5. Plugged females were anesthetized with Halothane inhalation followed by cervical dislocation. Uteri of pregnant females were dissected at E3.5, E4.5, E5.5, E10.5 and E18.5. Embryos at E10.5 were fixed in 10\% Formalin overnight before being weighted. Implantation sites at E4.5 and E5.5 were localized by i.v. injection of Evans blue dye ( $200 \mu \mathrm{l}, 1 \%$ in 1 xPBS, Sigma) ${ }^{16}$. The numbers of embryos initially implanted in both $\mathrm{LPA}_{3}$-deficient and WT/Het uteri were retrospectively calculated from E10.5 as following: At E10.5, embryos in an average of 1.2 implantation sites (out of 5.0 total) were absorbed in $\mathrm{LPA}_{3}$-deficient uteri, but embryos in only 0.09 implantation sites (out of 8.4 total) were absorbed in WT/Het uteri $\left(\mathrm{P}=1.7 \times 10^{-5}\right)$. With an average of 1.65 live embryos per implantation site in $\mathrm{LPA}_{3}$-deficient uteri and 1.0 live embryo per implantation site in WT/Het uteri at E10.5, the total number of embryos initially implanted should be 8.3 ((3.8 live +1.2 absorbed $) \mathrm{x} 1.65)$ in $\mathrm{LPA}_{3}$-deficient uteri and 8.4 ((8.31 live + 0.09 absorbed $\left.) \mathrm{x} 1.0\right) \mathrm{in} \mathrm{WT/}$ Het uteri.

\section{Prostaglandin Measurement}

Uteri from E3.5 WT or $\mathrm{LPA}_{3}$-deficient mice were immediately frozen and crushed in liquid nitrogen. Prostaglandins were extracted by ethyl acetate extraction method. The prostaglandin levels of each sample were determined using prostaglandin enzyme-linked immuno assay (EIA) kit (Cayman Chemical). PGI $_{2}$ was measured as 6-keto-PGF $1 \alpha$. 


\section{Prostaglandin Administration}

E3.5 $\mathrm{LPA}_{3}$-deficient females were i.p. injected with $100 \mu \mathrm{l}$ of vehicle (10\% ETOH with saline, as control) or $5 \mu \mathrm{g}$ cPGI and $5 \mu \mathrm{gGE}_{2}$ (Cayman Chemical, in $10 \%$ ETOH with saline) at 10:00 am and 6:00 pm. Implantation sites were detected using Evans blue dye at E4.5.

\section{Data Representation}

Data were expressed as Mean \pm SD. Statistical analyses were done using Student's $t$ test or $\chi^{2}$ test. The significant level was set at $\mathrm{P}<0.05$.

\section{Supplementary Material}

Refer to Web version on PubMed Central for supplementary material.

\section{Acknowledgements}

We thank Drs. F. Liu, S. Kupriyanov, R. Rivera, D. Herr, E. Nilsson, M. Murakami, Y. Kita, and B.C. Paria, Ms. S. Carlson and Q. Chen for technical assistance and insightful suggestions. This work was supported by grants from the National Institute of Mental Health to J.C. and J.J.A.C., the National Institute of Health to M.K.S, Swiss National Science Foundation to B.A., Program for Promotion of Fundamental Studies in Health Sciences of the Pharmaceuticals and Medical Devices Agency (PMDA) and grants-in-aid from the Ministry of Education, Science, Culture and Sports for the 21st Century Center of Excellence Program, Japan to J.A and H.A..

\section{References}

1. Dey SK, et al. Molecular cues to implantation. Endocr Rev 2004;25:341-73. [PubMed: 15180948]

2. Ishii I, Fukushima N, Ye X, Chun J. LYSOPHOSPHOLIPID RECEPTORS: Signaling and Biology. Annu Rev Biochem 2004;73:321-354. [PubMed: 15189145]

3. Bandoh K, et al. Molecular cloning and characterization of a novel human G-protein-coupled receptor, EDG7, for lysophosphatidic acid. J Biol Chem 1999;274:27776-85. [PubMed: 10488122]

4. Contos JJ, Chun J. The mouse lp(A3)/Edg7 lysophosphatidic acid receptor gene: genomic structure, chromosomal localization, and expression pattern. Gene 2001;267:243-53. [PubMed: 11313151]

5. Lim H, et al. Multiple female reproductive failures in cyclooxygenase 2-deficient mice. Cell 1997;91:197-208. [PubMed: 9346237]

6. Cleary-Goldman J, D'Alton M. Management of single fetal demise in a multiple gestation. Obstet Gynecol Surv 2004;59:285-98. [PubMed: 15024229]

7. Umstad MP, Gronow MJ. Multiple pregnancy: a modern epidemic? Med J Aust 2003;178:613-5. [PubMed: 12797847]

8. Daniel Y, et al. Analysis of 104 twin pregnancies conceived with assisted reproductive technologies and 193 spontaneously conceived twin pregnancies. Fertil Steril 2000;74:683-9. [PubMed: 11020507]

9. Wilcox AJ, Baird DD, Weinberg CR. Time of implantation of the conceptus and loss of pregnancy. N Engl J Med 1999;340:1796-9. [PubMed: 10362823]

10. Tokumura A, Fukuzawa K, Yamada S, Tsukatani H. Stimulatory effect of lysophosphatidic acids on uterine smooth muscles of non-pregant rats. Arch Int Pharmacodyn Ther 1980;245:74-83. [PubMed: 6902643]

11. Contos JJ, Fukushima N, Weiner JA, Kaushal D, Chun J. Requirement for the lpA1 lysophosphatidic acid receptor gene in normal suckling behavior. Proc Natl Acad Sci U S A 2000;97:13384-9. [PubMed: 11087877]

12. Contos JJ, et al. Characterization of lpa(2) (Edg4) and lpa(1)/lpa(2) (Edg2/Edg4) lysophosphatidic acid receptor knockout mice: signaling deficits without obvious phenotypic abnormality attributable to lpa(2). Mol Cell Biol 2002;22:6921-9. [PubMed: 12215548]

13. Kingsbury MA, Rehen SK, Contos JJ, Higgins CM, Chun J. Non-proliferative effects of lysophosphatidic acid enhance cortical growth and folding. Nat Neurosci 2003;6:1292-9. [PubMed: 14625558] 
14. Inoue $\mathrm{M}$, et al. Initiation of neuropathic pain requires lysophosphatidic acid receptor signaling. Nat Med 2004;10:712-8. [PubMed: 15195086]

15. Noguchi K, Ishii S, Shimizu T. Identification of p2y9/GPR23 as a novel G protein-coupled receptor for Lysophosphatidic acid, structurally distant from the Edg family. J Biol Chem. 2003

16. Paria BC, Huet-Hudson YM, Dey SK. Blastocyst's state of activity determines the "window" of implantation in the receptive mouse uterus. Proc Natl Acad Sci U S A 1993;90:10159-62. [PubMed: 8234270]

17. Kennedy TG. Evidence for a role for prosaglandins in the initiation of blastocyst implantation in the rat. Biol Reprod 1977;16:286-91. [PubMed: 843558]

18. Kinoshita K, et al. Involvement of prostaglandins in implantation in the pregnant mouse. Adv Prostaglandin Thromboxane Leukot Res 1985;15:605-7. [PubMed: 2936179]

19. Song H, et al. Cytosolic phospholipase A2alpha is crucial [correction of A2alpha deficiency is crucial] for 'on-time' embryo implantation that directs subsequent development. Development 2002;129:2879-89. [PubMed: 12050136]

20. Frenkian M, Segond N, Pidoux E, Cohen R, Jullienne A. Indomethacin, a COX inhibitor, enhances 15-PGDH and decreases human tumoral C cells proliferation. Prostaglandins 2001;65:11-20. [PubMed: 11334638]

21. Reese J, Brown N, Paria BC, Morrow J, Dey SK. COX-2 compensation in the uterus of COX-1 deficient mice during the pre-implantation period. Mol Cell Endocrinol 1999;150:23-31. [PubMed: 10411296]

22. Sibilia M, Wagner EF. Strain-dependent epithelial defects in mice lacking the EGF receptor. Science 1995;269:234-8. [PubMed: 7618085]

23. Wang $\mathrm{H}$, et al. Rescue of female infertility from the loss of cyclooxygenase-2 by compensatory upregulation of cyclooxygenase-1 is a function of genetic makeup. J Biol Chem 2004;279:10649-58. [PubMed: 14701858]

24. Lim H, et al. Cyclo-oxygenase-2-derived prostacyclin mediates embryo implantation in the mouse via PPARdelta. Genes Dev 1999;13:1561-74. [PubMed: 10385625]

25. Yang ZM, et al. Potential sites of prostaglandin actions in the periimplantation mouse uterus: differential expression and regulation of prostaglandin receptor genes. Biol Reprod 1997;56:368-79. [PubMed: 9116135]

26. Paria BC, Song H, Dey SK. Implantation: molecular basis of embryo-uterine dialogue. Int J Dev Biol 2001;45:597-605. [PubMed: 11417904]

27. Gabardi S, Cerio J. Future immunosuppressive agents in solid-organ transplantation. Prog Transplant 2004;14:148-56. [PubMed: 15264459]

28. Ishii I, et al. Marked perinatal lethality and cellular signaling deficits in mice null for the two sphingosine 1-phosphate (S1P) receptors, S1P(2)/LP(B2)/EDG-5 and S1P(3)/LP(B3)/EDG-3. J Biol Chem 2002;277:25152-9. [PubMed: 12006579]

29. Hama K, et al. Lysophosphatidic acid and autotaxin stimulate cell motility of neoplastic and nonneoplastic cells through LPA1. J Biol Chem 2004;279:17634-9. [PubMed: 14744855]

30. Ishii I, et al. Selective loss of sphingosine 1-phosphate signaling with no obvious phenotypic abnormality in mice lacking its $\mathrm{G}$ protein-coupled receptor, LP(B3)/EDG-3. J Biol Chem 2001;276:33697-704. [PubMed: 11443127] 

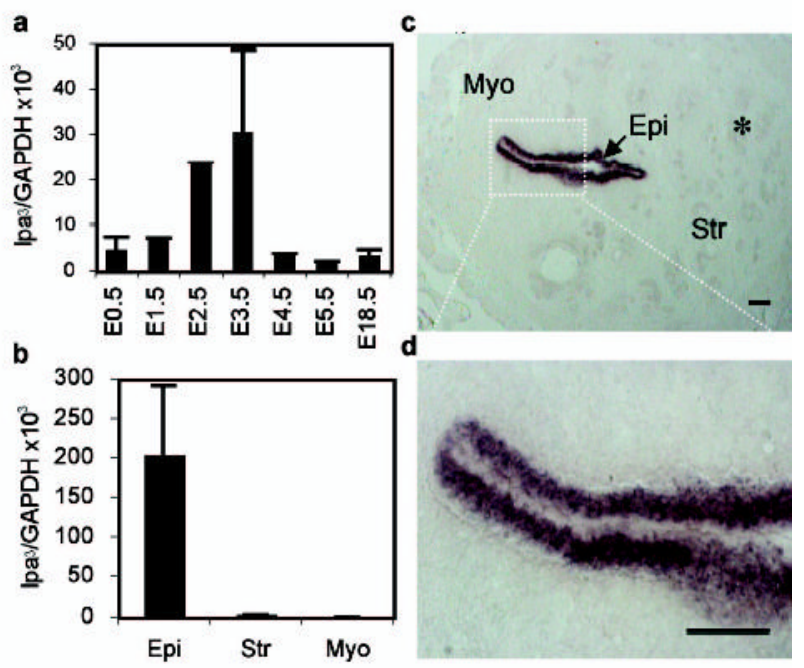

e

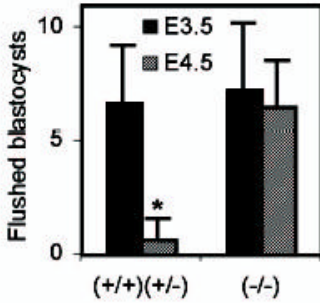

$\mathbf{f}$

g

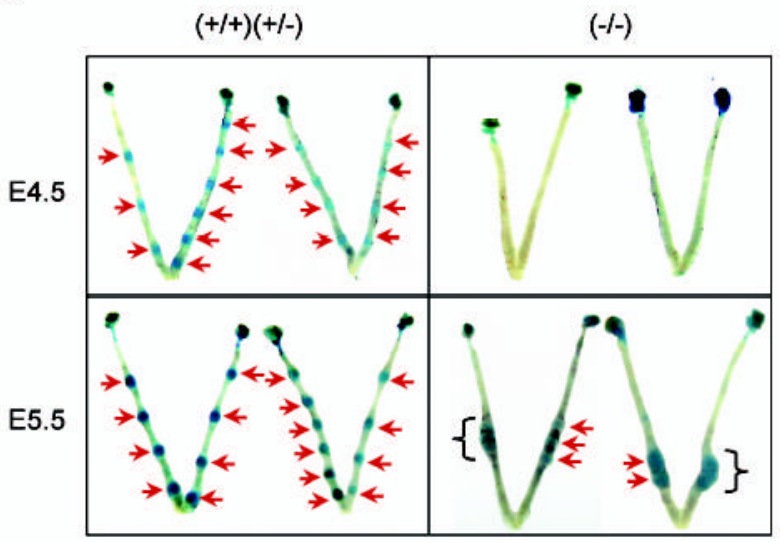

Figure 1.

$\mathrm{LPA}_{3}$ mRNA expression in WT uterus and effects of $\mathrm{LPA}_{3}$-deficiency on implantation. a,b. Quantification of uterine $\mathrm{LPA}_{3}$ mRNA during pregnancy and in E3.5 luminal endometrial epithelium (Epi), stroma (Str), and myometrium (Myo). c,d. In situ localization of $\mathrm{LPA}_{3}$ in E3.5 WT uterus. *. Glandular endometrial epithelium. Scale bars $=100 \mu \mathrm{m}$. e. Flushed blastocysts from E3.5 and E4.5 uteri. f,g. Number and location of implantation sites at E4.5 and E5.5 uteri. Blue bands (arrows): implantation sites; Brackets: clustered implantation sites. $* \mathrm{P}<0.001$. In all figures, error bars are standard deviations, $(+/+),(+/-)$, and $(-/-)$ represent WT, Het, and $\mathrm{LPA}_{3}$-deficient mice, respectively. 
a
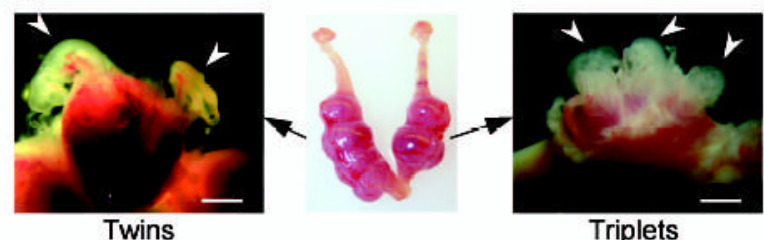

Triplets

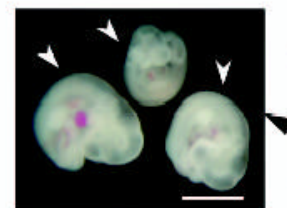

Triplets

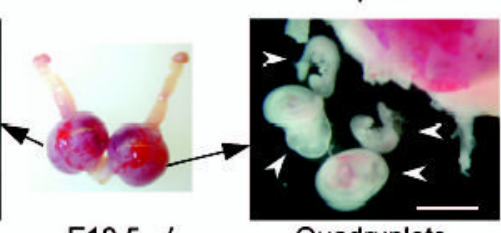

$\mathrm{E} 10.5,-/-$

Quadruplets

b
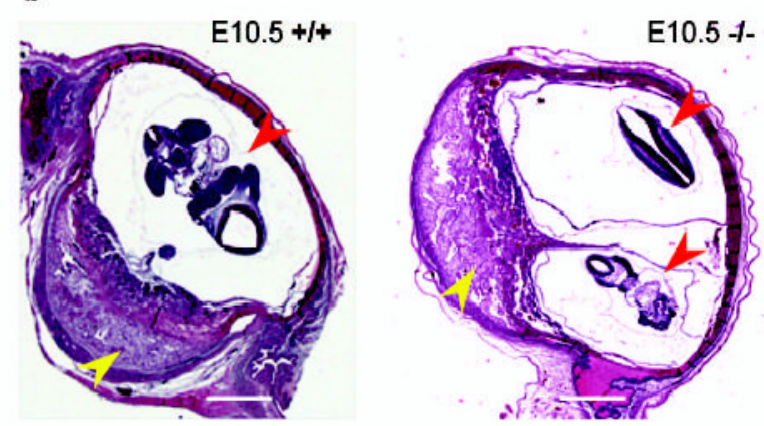

c
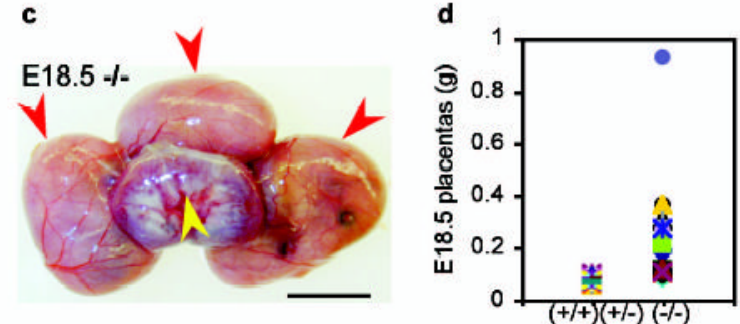

Figure 2.

Multiple embryos at individual implantation sites and placental hypertrophy in $\mathrm{LPA}_{3}$-deficient uteri. a. Samples of multiple embryos at individual implantation sites at E10.5. White arrowheads: embryos. Scale bars: $2 \mathrm{~mm}$. b. Cross-sections of E10.5 uteri revealing two less developed embryos sharing one placenta in a $\mathrm{LPA}_{3}$-deficient uterus. Scale bars: $1 \mathrm{~mm}$. c. A placenta shared by three embryos at E18.5 in a LPA 3 -deficient uterus. Scale bar: $8 \mathrm{~mm}$. b and c, red arrowheads: embryos; yellow arrowheads: placentas. d. Weight of placentas at E18.5. 
a

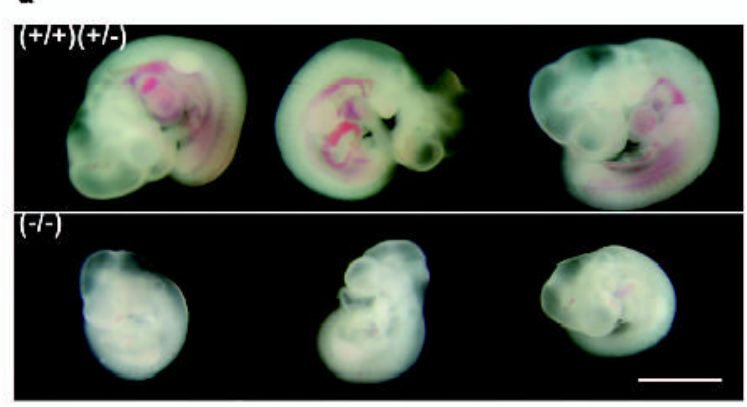

b

C
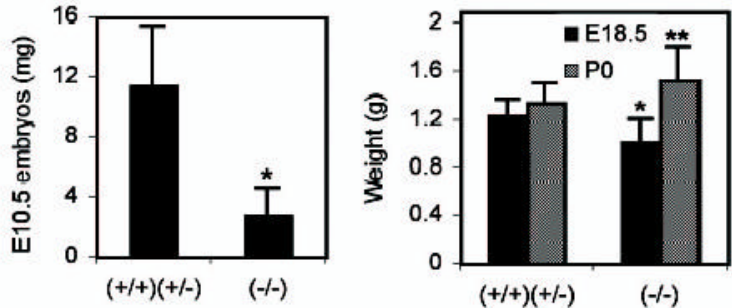

d

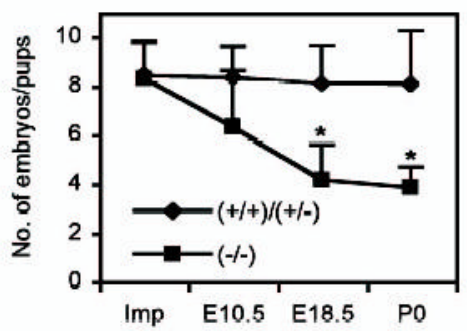

Figure 3.

Delayed postimplantational development of embryos and increased embryonic death in LPA $_{3}$-deficient uteri. a. Representative embryos from E10.5 uteri. Scale bar: $2 \mathrm{~mm}$. b. E10.5 embryo weight. c. Weights of E18.5 embryos and P0 pups. d. The average numbers of embryos implanted (Imp), at E10.5 and E18.5, and P0 pups. The numbers of embryos implanted were calculated as described in Material and Methods. $* \mathrm{P}<0.001, * * \mathrm{P}<0.05$. 


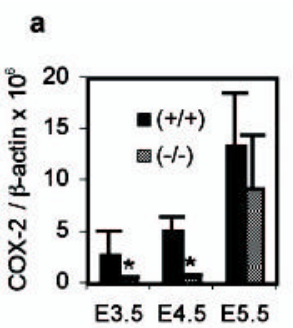

b

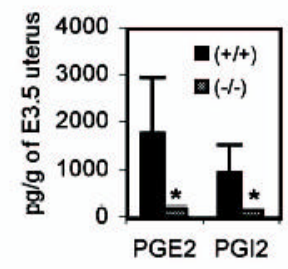

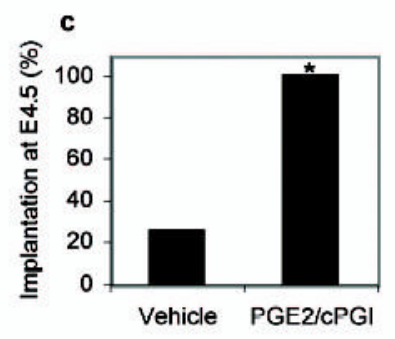

d

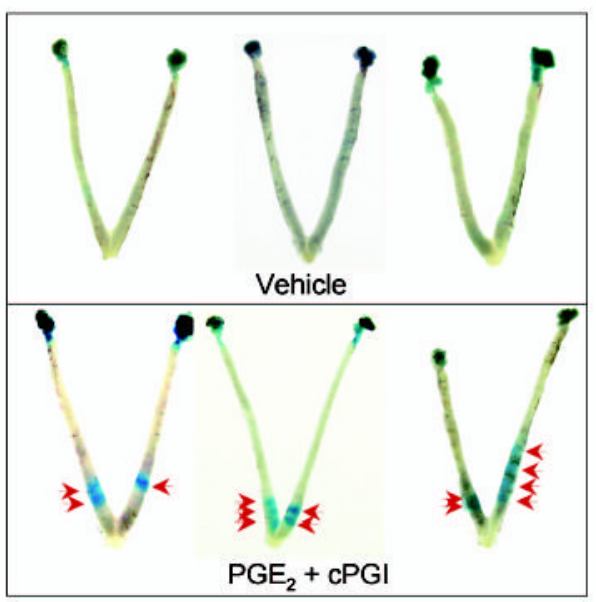

Figure 4.

Reduced COX-2 mRNA and prostaglandin levels in $\mathrm{LPA}_{3}$-deficient uteri, and exogenous prostaglandin rescue of delayed implantation. a. Expression of COX-2 during early pregnancy in WT and $\mathrm{LPA}_{3}$-deficient uteri. ${ }^{*} \mathrm{P}<0.05$. b. Reduced $\mathrm{PGE}_{2}$ and $\mathrm{PGI}_{2}$ levels in $\mathrm{E} 3.5 \mathrm{LPA}_{3}$ deficient uteri. ${ }^{*} \mathrm{P}<0.05$. c. Significantly increased percentage of $\mathrm{LPA}_{3}$-deficient females showing on-time implantation upon $\mathrm{PGE}_{2}$ and cPGI (a stable $\mathrm{PGE}_{2}$ analog) treatment at E3.5. $* \mathrm{P}=0.003$. d. Images of E4.5 $\mathrm{LPA}_{3}$-deficient uteri with or without prostaglandin treatment. Red arrows: implantation sites. Supplementary Figure S1a, S1b, S1c 